\title{
Calculation of excitation energies from the CC2 linear response theory using Cholesky decomposition
}

Pablo Baudin' , José Sánchez Marín, Inmaculada García Cuesta, and Alfredo M. J. Sánchez de Merás’

Citation: The Journal of Chemical Physics 140, 104111 (2014); doi: 10.1063/1.4867270

View online: http://dx.doi.org/10.1063/1.4867270

View Table of Contents: http://aip.scitation.org/toc/jcp/140/10

Published by the American Institute of Physics

\section{Articles you may be interested in}

Transition moments and excited-state first-order properties in the coupled-cluster model CC2 using the resolution-of-the-identity approximation

The Journal of Chemical Physics 117, 6939 (2002); 10.1063/1.1506918

CC2 excitation energy calculations on large molecules using the resolution of the identity approximation

The Journal of Chemical Physics 113, 5154 (2016); 10.1063/1.1290013

The closed-shell coupled cluster single and double excitation (CCSD) model for the description of electron correlation. A comparison with configuration interaction (CISD) results

The Journal of Chemical Physics 86, 2881 (1998); 10.1063/1.452039

LoFEx - A local framework for calculating excitation energies: Illustrations using RI-CC2 linear response theory The Journal of Chemical Physics 144, 224106 (2016); 10.1063/1.4953360

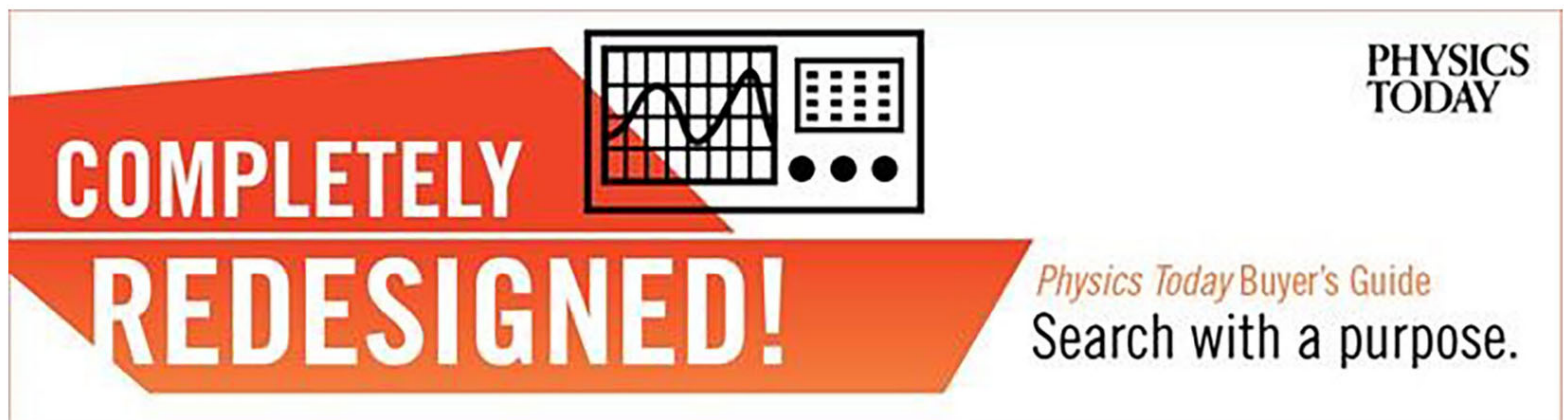




\title{
Calculation of excitation energies from the CC2 linear response theory using Cholesky decomposition
}

\author{
Pablo Baudin, ${ }^{1,2, a)}$ José Sánchez Marín, ${ }^{1}$ Inmaculada García Cuesta, ${ }^{1}$ \\ and Alfredo M. J. Sánchez de Merás ${ }^{1, b)}$ \\ ${ }^{1}$ Institute of Molecular Science, University of Valencia, Valencia, ES-46071, Spain \\ ${ }^{2}$ qLEAP - Center for Theoretical Chemistry, Department of Chemistry, Aarhus University, \\ Langelandsgade 140, DK-8000 Aarhus C, Denmark
}

(Received 25 October 2013; accepted 18 February 2014; published online 12 March 2014)

\begin{abstract}
A new implementation of the approximate coupled cluster singles and doubles CC2 linear response model is reported. It employs a Cholesky decomposition of the two-electron integrals that significantly reduces the computational cost and the storage requirements of the method compared to standard implementations. Our algorithm also exploits a partitioning form of the CC2 equations which reduces the dimension of the problem and avoids the storage of doubles amplitudes. We present calculation of excitation energies of benzene using a hierarchy of basis sets and compare the results with conventional $\mathrm{CC} 2$ calculations. The reduction of the scaling is evaluated as well as the effect of the Cholesky decomposition parameter on the quality of the results. The new algorithm is used to perform an extrapolation to complete basis set investigation on the spectroscopically interesting benzylallene conformers. A set of calculations on medium-sized molecules is carried out to check the dependence of the accuracy of the results on the decomposition thresholds. Moreover, CC2 singlet excitation energies of the free base porphin are also presented. ( 2014 AIP Publishing LLC. [http://dx.doi.org/10.1063/1.4867270]
\end{abstract}

\section{INTRODUCTION}

Quantum mechanics has proved to be an indispensable tool for the description of molecular systems and their chemical properties. Decades of theoretical research have provided chemists with a set of very powerful methods. Among them, coupled cluster theory is universally recognized as the method of choice for small to medium size molecules. However, its computational cost forces researcher to use less expensive and, as a consequence, less accurate theories for the treatment of larger systems.

Since the beginning of the new century, quantum chemistry is changing. Considerable efforts are now oriented in reducing the scaling of expensive methods like coupled cluster and a lot of progress has been made. Some groups develop local approximations, ${ }^{1-4}$ others focus on the approximation of the two-electron integrals ${ }^{5-7}$ and totally new algorithm are developed to be well adapted to new technologies (massive parallelization, ${ }^{8,9}$ graphics processing units, ${ }^{10,11}$ etc.). In particular, the Cholesky decomposition arises as an efficient tool for the reduction of computational efforts as well as storage requirements.

Computation of excited states and frequency-dependent properties is a very important and active field in quantum chemistry. Indeed, the comprehension of electronic structure of molecular systems is often closely related to the investigation of their spectroscopic properties. Moreover, since the interpretation of experimental data can be sometimes very

\footnotetext{
a)baudin.pablo@gmail.com

b) sanchez@uv.es
}

complicated, computational chemistry and especially the calculation of frequency-dependent properties like excitation energies can be very useful. Therefore, it is very important to reduce the scaling of the most accurate methods and thus extend their applicability to large molecules. ${ }^{12}$

The goal of this work is to apply the Cholesky decomposition (CD) of the two-electron integral matrix to the calculation of excitation energies. For this purpose, we chose a CC2 ground state wave-function together with a linear response function for the calculation of excitation energies. Some successful implementations of the Cholesky decomposition to the CC2 model have already been done and we hope to considerably extend the applicability of the method for the computation of excitation energies. ${ }^{13}$

The CC2 model is the simplest model of the coupled cluster hierarchy that recovers some dynamical correlation. Moreover, this model was especially design for the calculation of frequency-dependent properties as single excitations are treated as zero order parameters. ${ }^{14}$ Its computational cost is similar to that of MP2 with the additional drawback of being iterative but the $\mathrm{CC} 2$ model constitutes a good compromise between highly correlated coupled cluster model like CCSDT, CC3, CCSDR(3), and non-correlated model with low computational cost.

The CC2 linear response scheme provides reasonably good vertical electronic excitation energies for singleconfigurational system, such that the typical error is of the order of $0.2-0.3 \mathrm{eV} .^{15,16}$ The great advantage of the Cholesky decomposition is its ability to control the introduced error via a single parameter. ${ }^{6}$ Therefore, the conventional CC2 results should be in principle recoverable. 
This paper is organized as follows: In Sec. II, important theoretical concepts are exposed regarding the Cholesky decomposition and the CC2 linear response model for the calculation of excitation energies. Section III contains the most important points of our implementation for the calculation of excitation energies and the optimization process. In Sec. IV, we present calculation of vertical excitation energies on several molecular systems. The results are analyzed in order to evaluate the quality of the implementation. Some concluding remarks are given in Sec. V.

\section{THEORY}

In this section, we survey how the Cholesky decomposition can be efficiently applied to the two-electron integrals. Then we expose the basis of the CC2 model and present the $\mathrm{CC} 2$ linear response equations from which excitation energies can be extracted.

\section{A. Cholesky decomposition of the two-electron integral}

Evaluation and storage of the two-electron integrals are well known to be one of the bottlenecks of most of quantum chemical methods. It is, therefore, important to develop new algorithms that reduce both the computational cost and the storage requirements of the two-electron integrals. The Cholesky decomposition is one of the efficient tools that allow to attain this goal. ${ }^{17,18}$

The efficiency of the Cholesky decomposition comes from its ability to remove linear dependencies and to control the introduced error via a single parameter. CD is often compared to the resolution of the identity approximation (RI) and it has been shown that they constitute two different formulations of the same mathematical operation. Among the most important differences, we remark that $\mathrm{CD}$ is an iterative process controlled via a single parameter while RI is non-iterative but requires a pre-optimized auxiliary basis set and the error cannot be directly controlled. ${ }^{6,18}$

The Cholesky decomposition of the atomic orbital (AO) two-electron integrals is given by ${ }^{18}$

$$
(\alpha \beta \mid \gamma \delta)=\sum_{J=1}^{K} L_{\alpha \beta}^{J} L_{\gamma \delta}^{J},
$$

where Greek letters are used for atomic orbitals and $K$ is the number of Cholesky vectors $L^{J}$. Reduction of computational cost arises when the number of Cholesky vectors is significantly lower than the full dimension $N(N+1) / 2$, where $N$ is the number of atomic orbitals. If the decomposition is complete, $K$ corresponds to the rank of the matrix, i.e., the full dimension minus the number of linear dependencies. The number of Cholesky vectors can be further reduced by stopping the decomposition based on a single parameter, $\tau$. In practice, the CD threshold should be chosen between $10^{-10}$ and $10^{-5}$, which allows to discard negligible integrals without significant loss of accuracy. To this end, we may express CD as an iterative process,

$$
\mathbf{M}^{(n+1)}=\mathbf{M}^{(n)}-L^{J_{n}}\left(L^{J_{n}}\right)^{T},
$$

where $\mathbf{M}^{(0)}=(\alpha \beta \mid \gamma \delta)$ and the Cholesky vectors $L^{J_{n}}$ are given by

$$
L_{i}^{J_{n}}=\frac{\mathbf{M}_{i J_{n}}^{(n)}}{\sqrt{\mathbf{M}_{J_{n} J_{n}}^{(n)}}} .
$$

Obviously, $\mathbf{M}_{J_{n} J_{n}}^{(n)}$ should be strictly positive and in general it is chosen to be the maximum diagonal element. ${ }^{17} \mathrm{~A}$ simplified implementation of $\mathrm{CD}$ can be summarized as follows:

1. Precalculate full diagonal $D_{i}^{(0)}=\mathbf{M}_{i i}^{(0)}$.

2. Find the largest diagonal element, $D_{\max }^{(i)}$. If $D_{\max }^{(i)}<\tau$, exit.

3. Cauchy-Schwarz screening: Get set of indices $\{p\}$ to be computed in the column of the diagonal elements. $r \in\{p\}$ if $\sqrt{D_{\max }^{(i)} D_{r}}>\tau$.

4. Calculate matrix elements $M_{p q}$ and get the associated Cholesky vectors.

5. Update diagonal by subtracting the contribution from the new vectors and go back to (2).

For efficiency reasons, the whole algorithm is driven by shells of atomic basis functions. More details can be found in Ref. 18.

\section{B. CC2 model and excitation energies}

The CC2 model was introduced by Christiansen et al. in Ref. 14 as an approximation to the CCSD model based on perturbation theory. The amplitude equations are given by

$$
\begin{aligned}
& \Omega_{\mu_{1}}=\left\langle\mu_{1}\left|\hat{H}+\left[\hat{H}, T_{2}\right]\right| \mathrm{HF}\right\rangle=0, \\
& \Omega_{\mu_{2}}=\left\langle\mu_{2}\left|\hat{H}+\left[F, T_{2}\right]\right| \mathrm{HF}\right\rangle=0,
\end{aligned}
$$

where $\left\{|\mathrm{HF}\rangle,\left|\mu_{1}\right\rangle,\left|\mu_{2}\right\rangle\right\}$ denote the Hartree-Fock ground state and the set of single and double excitation manifolds. $F$ is the Fock operator and the $\Omega$ is called the coupled cluster vector function. $\hat{H}$ is a similarity transformed Hamiltonian,

$$
\hat{H}=e^{-T_{1}} H e^{T_{1}},
$$

where we used $T_{i}=\sum_{\mu_{i}} t_{\mu_{i}} \tau_{\mu_{i}}$. Keeping the Hamiltonian in the form of Eq. (6) allows to treat the singles as zero order parameters in a many body perturbation theory (MBPT) formulation of the $\mathrm{CC} 2$ model with a fluctuation potential defined as $U=H-F$. It is important to provide a good description of the single excitations as they play an important role in the description of frequency-dependent properties and in particular for the calculation of excitation energies. ${ }^{19}$

The CC2 model was first implemented as a special case of the CCSD algorithm without taking particular advantage of the simplified form of the $\mathrm{CC} 2$ equations. ${ }^{20}$ A new implementation strategy has been proposed by Hättig et al. ${ }^{21-24}$ in which the doubles-which have a simple form in the CC2 model - are computed on-the-fly and directly contracted to form three-index intermediates that are stored on disk. In order to be reliable, such an implementation requires a factorization of the two-electron integrals as provided by the RI or 
the CD. This doubles-direct algorithm has been described in detailed in Ref. 21 using RI both for the CC2 wave-function optimization and for calculation of excitation energies. In the following, we briefly remind how to compute excitation energies using the linear response function and a partitioning form of the $\mathrm{CC} 2$ equations.

In the response function scheme, a time-independent Hamiltonian is perturbed by a time-dependent potential $\hat{V}$. There are different possibilities to derive expression for the coupled cluster response function. One of them is based on a quasi-energy Lagrangian. ${ }^{20,25,26}$ The $\mathrm{CC} 2$ quasi-energy
Lagrangian is given by

$$
\begin{aligned}
\mathcal{L}= & \left\langle\mathrm{HF}\left|H e^{T_{1}+T_{2}}\right| \mathrm{HF}\right\rangle \\
& +\sum_{\mu_{1}} \bar{t}_{\mu_{1}}\left(\left\langle\mu_{1}\left|\hat{H}+\left[\hat{H}, T_{2}\right]\right| \mathrm{HF}\right\rangle-i \dot{t}_{\mu_{1}}\right) \\
& +\sum_{\mu_{2}} \bar{t}_{\mu_{2}}\left(\left\langle\mu_{2}\left|\hat{H}+\left[F+\hat{V}, T_{2}\right]\right| \mathrm{HF}\right\rangle-i \dot{t}_{\mu_{2}}\right) .
\end{aligned}
$$

From this Lagrangian, the $\mathrm{CC} 2$ linear response can be derived following Refs. 20 and 26, and the poles of the linear response arise at the eigenvalues of the CC2 Jacobian matrix,

$$
\mathbf{A}_{\mu_{i} \nu_{j}}=\frac{\partial \Omega_{\mu_{i}}}{\partial t_{v_{j}}}=\left(\begin{array}{cc}
\left\langle\mu_{1}\left|\left[\hat{H}, \tau_{\nu_{1}}\right]+\left[\left[\hat{H}, \tau_{\nu_{1}}\right], T_{2}\right]\right| \mathrm{HF}\right\rangle & \left\langle\mu_{1}\left|\left[\hat{H}, \tau_{v_{2}}\right]\right| \mathrm{HF}\right\rangle \\
\left\langle\mu_{2}\left|\left[\hat{H}, \tau_{\nu_{1}}\right]\right| \mathrm{HF}\right\rangle & \delta_{\mu_{2} v_{2}} \epsilon_{v_{2}}
\end{array}\right) .
$$

Hence, finding excitation energies is equivalent to find the eigenvalues of the $\mathrm{CC} 2$ Jacobian by solving the eigenvalue problem, ${ }^{14}$

$$
\mathbf{A} b_{k}=\omega_{k} b_{k} \text {. }
$$

Using the fact that the doubles-doubles block $\mathbf{A}_{\mu_{2} v_{2}}$ is already diagonal, we can reduce the dimension of the eigenvalue problem (9). In the doubles-doubles block, we have $\epsilon_{a i b j}=\epsilon_{a}-\epsilon_{i}$ $+\epsilon_{b}-\epsilon_{j}$, where $\epsilon_{p}$ corresponds to the SCF energy of orbital $p$. All the information about excitation energies can now be extracted from the solutions of the pseudo-eigenvalue problem,

$$
\mathbf{A}_{\mu_{1} \nu_{1}}^{\mathrm{eff}}(\omega) b_{k}=\left[\mathbf{A}_{\mu_{1} \nu_{1}}-\frac{\mathbf{A}_{\mu_{1} \gamma_{2}} \mathbf{A}_{\gamma_{2} \nu_{1}}}{\epsilon_{\gamma_{2}}-\omega}\right] b_{k}=\omega_{k} b_{k},
$$

which has the dimension of the single excitation manifold but the effective Jacobian on the left hand side of Eq. (10) is now dependent on its eigenvalues, which implies that the solutions have to be found until self-consistency is reached. ${ }^{21}$

\section{IMPLEMENTATION}

In this section, we describe our implementation of the CD-CC2 algorithm for calculation of excitation energies. In the first part, we present an adaptation of the doublesdirect algorithm to $\mathrm{CD}$ while the second part is dedicated to the method used to solve the pseudo-eigenvalue problem (Eq. (10)).

\section{A. Construction of the linear transformed vector}

For the implementation, we follow the strategy devised by Hättig et al. ${ }^{21}$ which was adapted by Pedersen $e t$ al. ${ }^{13}$ to use CD instead of RI. In this subsection, we briefly summarize their main findings and more details can be found in Refs. 21 and 13 .

Computation of excitation energies requires some calculations to be done. In particular, a SCF optimization provides orbital energies $\epsilon_{p}$ and transformation coefficients $\mathbf{C}$. Moreover, we assume the two-electron integrals to be already decomposed in terms of Cholesky vectors in atomic basis and stored on disk in an efficient manner. Finally, a CC2 optimization of the wave-function should be done providing the set of single amplitudes $\left\{t_{\mu_{1}}\right\}$.

The most demanding parts of the algorithm involve computation and storage of a particular class of intermediates of the general form,

$$
Y_{a i}^{J}=\sum_{b j} X_{i j}^{a b} \hat{L}_{j b}^{J},
$$

where $\hat{L}_{j b}^{J}$ is a transformed Cholesky vector given by

$$
\begin{gathered}
\hat{L}_{j b}^{J}=\sum_{\alpha \beta} \Lambda_{\alpha j}^{p} L_{\alpha \beta}^{J} \Lambda_{\beta b}^{h}, \\
\boldsymbol{\Lambda}^{p}=\mathbf{C}\left(\mathbf{1}-\boldsymbol{t}_{1}^{T}\right), \\
\boldsymbol{\Lambda}^{h}=\mathbf{C}\left(\mathbf{1}+\boldsymbol{t}_{1}\right),
\end{gathered}
$$

and $X_{i j}^{a b}$ corresponds either to the symmetrized double amplitudes,

$$
\tilde{f}_{i j}^{a b}=\left(1+\delta_{i j} \delta_{a b}\right)\left(2 t_{i j}^{a b}-t_{j i}^{b a}\right)=\frac{2(a i \hat{\mid} b j)-(b i \hat{\mid} a j)}{\epsilon_{i}-\epsilon_{a}+\epsilon_{j}-\epsilon_{b}},
$$

where,

$$
(a i \hat{\mid} b j)=\sum_{J} \hat{L}_{a i}^{J} \hat{L}_{b j}^{J},
$$

or to the four-index auxiliary vector,

$$
\bar{b}_{i j}^{a b}=\frac{2(a i \overline{\mid} b j)-(b i \overline{\mid} a j)}{\epsilon_{i}-\epsilon_{a}+\epsilon_{j}-\epsilon_{b}+\omega},
$$

with,

$$
\begin{aligned}
& (a i \overline{\rceil} b j)=\sum_{J} \bar{L}_{a i}^{J} \hat{L}_{b j}^{J}+\sum_{J} \bar{L}_{b j}^{J} \hat{L}_{a i}^{J}, \\
& \bar{L}_{a i}^{J}=\sum_{\alpha \beta}\left(\bar{\Lambda}_{\alpha a}^{p} \Lambda_{\beta i}^{h}+\Lambda_{\alpha a}^{p} \bar{\Lambda}_{\beta i}^{h}\right) L_{\alpha \beta}^{J} .
\end{aligned}
$$

The perturbed transformation matrices $\bar{\Lambda}^{p}$ and $\bar{\Lambda}^{h}$ are defined as $\bar{\Lambda}^{p}=-\mathbf{C} \mathbf{b}_{\mu_{1}}^{T}$ and $\bar{\Lambda}^{h}=\mathbf{C} \mathbf{b}_{\mu_{1}}$. 
Computation of these $Y$-intermediates scales as $O^{2} V^{2} \mathrm{~K}$ with $O$ the number of occupied orbitals, $V$ the number of virtual orbitals, and $K$ the number of Cholesky vectors. ${ }^{13}$ The most important steps of the CD-CC2 algorithm can be summarized as follows:

1. Transform Cholesky vectors from AO to MO basis using $\boldsymbol{\Lambda}$ transformation matrices (Eq. (12)). Store them on disk.

2. Calculate intermediates $E_{a b}^{(1)}$ and $E_{i j}^{(2)}$ via $Y$ intermediates calculated using $X_{i j}^{a b}=\tilde{t}_{i j}^{a b}$. Store them on disk.

3. Generate trial frequencies $\omega_{k}$ and vectors $b_{k}$ from SCF orbitals.

4. Enter optimization loop:

(a) Calculate $\sigma_{a j}^{0}$ using $E$-intermediates and $b_{k}$ vectors.

(b) Calculate $\sigma_{a i}^{J}$ and $\sigma_{a i}^{I 2}$.

(c) Transform perturbed Cholesky vectors (Eq. (19)) and store them on disk.

(d) Calculate $Y$-intermediates using $X_{i j}^{a b}=\bar{b}_{i j}^{a b}$ and store them on disk. $\bar{b}_{i j}^{a b}$ is also contracted with the Fock matrix to get the contribution in $\sigma_{a i}^{I 1}$.

(e) Calculate $\sigma_{a i}^{G}$ and $\sigma_{a i}^{H}$ using $Y$-intermediates from disk.

The explicit expressions of intermediates used in the pseudo-code above are presented in Table I.

\section{B. Resolution of the pseudo-eigenvalue problem}

As stated before, the partitioning of the eigenvalue problem implies the optimization process to be done until selfconsistency is reached. In order to do so we used a modified Davidson algorithm that allows to compute only a few eigenvalues of a large matrix. ${ }^{27}$

The first batches of linear transformed vectors are built using a guessed eigenvalue (typically a difference of SCF orbital energies). When the Davidson process converges the new eigenvalue is compared to the guessed one and if the difference is too large, a new Davidson optimization is started using the last optimized frequency to compute the linear transformed vectors and the last Ritz vectors as subspace of trial eigenvectors. The Ritz vectors are given by

$$
\xi^{i}=\mathbf{B x}^{i},
$$

TABLE I. Explicit expressions of the linear transformed vector. ${ }^{21}$

$$
\begin{aligned}
& \sigma_{a i}(\omega, b)=\sigma_{a i}^{0}+\sigma_{a i}^{G}+\sigma_{a i}^{H}+\sigma_{a i}^{I 1}+\sigma_{a i}^{I 2}+\sigma_{a i}^{J}, \\
& \sigma_{a i}^{0}=\sum_{b} E_{a b}^{(1)} b_{b i}-\sum_{j} b_{a j} E_{j i}^{(2)}, \\
& \sigma_{a i}^{G}=\sum_{d l c} \bar{b}_{i l}^{c d}(l d \hat{\mid} a c), \\
& \sigma_{a i}^{H}=-\sum_{d l k} \bar{b}_{k l}^{a d}(l d \hat{\mid} k i), \\
& \sigma_{a i}^{I 1}=\sum_{d l} \bar{b}_{i l}^{a d} \hat{F}_{l d}, \\
& \sigma_{a i}^{I 2}=\sum_{d l} \tilde{t}_{i l}^{a d} \bar{F}_{l d}, \\
& \sigma_{a i}^{J}=\sum_{c k}[2(a i \mid k c)-(a c \hat{\mid} k i)] b_{c k}, \\
& E_{a b}^{(1)}=\hat{F}_{a b}-\sum_{d l k} \tilde{t}_{k l}^{a d}(l d \hat{\mid} k b), \\
& E_{i j}^{(2)}=\hat{F}_{i j}+\sum_{d l c} \tilde{t}_{i l}^{c d}(l d \hat{\mid} j c), \\
& \bar{F}_{l d}=\sum_{c k}[2(l d \hat{\mid} k c)-(l c \hat{\mid} k d)] b_{c k} .
\end{aligned}
$$

where the trial eigenvectors form the column vectors of $\mathbf{B}$ and $\mathbf{x}^{i}$ is the eigenvector $i$ solution of the reduced problem. This whole process is continued until self-consistency. The modified Davidson method is now a one root optimization process as the only valuable excitation energy from the Davidson optimization is the one that corresponds to the frequency used to build the linear transformed vectors. Therefore, we will have to do a self-consistent optimization for each required root. However, the method can be adapted to those conditions. The threshold used to evaluate the quality of the residual has to be an evaluation of self-consistency, $\tau=\left|\omega_{\text {input }}-\omega^{j}\right|$. In this way the Davidson process converges faster when we are far from self-consistency, which avoids useless calculations. Another important improvement is to start a new Davidson optimization with the last Ritz vectors even when starting a new self-consistent process. In general, they constitute a good approximation to eigenvectors and will make the Davidson process to converge much faster. ${ }^{27}$ Further improvements can be achieved using optimized coupled cluster singles (CCS) eigenvectors as starting guess for the $\mathrm{CC} 2$ algorithm.

Finally, it is important to notice that in this algorithm, each optimized root is the result of the diagonalization of a different Jacobian matrix. Indeed, the input frequency is different for each root. As a consequence, the excited states implicitly involved can theoretically interact between them. However, the small differences between the excitation energies of different roots and the intrinsic error of the $\mathrm{CC} 2$ method make those differences not notable. As a matter of fact, the results obtained using a $\mathrm{CD}-\mathrm{CC} 2$ excitation energy algorithm show no differences compared to excitation energies calculated by diagonalization of the full non-effective CC2 Jacobian matrix (Sec. IV A).

Alternatively (as done in Ref. 21), it is also possible to use a direct inversion of the iterative subspace (DIIS) extrapolation analogous to the one normally used to solve the nonlinear equations determining the coupled cluster ground state. Comparing to the previously discussed modification of the Davidson algorithm, DIIS extrapolation has the advantages of being faster and a single-root method. On the other side, it is less robust than Davidson procedure and, in fact, DIIS extrapolation can diverge if the initial guess is not close enough to the converged solution. This drawback can be solved ${ }^{21}$ by combining it with a preconditioning Davidson process in which the Jacobian dependence on the excitation energy is discarded. In Sec. IV C, we compare these two approaches.

\section{APPLICATIONS}

In order to test our new implementation of CD-CC2 linear response theory for the calculation of excitation energies, we first apply it to the well known benzene molecule using large basis set and compare the results with different CC2 implementations. Then we propose an extrapolation to the complete basis set (CBS) of the lowest excitation energies of benzylallene which has interesting spectroscopic properties. In Sec. IV C, we study the dependence of the accuracy of the excitation energies on the decomposition thresholds and compare the two ways of solving the pseudo-eigenvalue problem. Finally, we present an investigation of the free base 
porphin electronic excitation spectrum which could be use to investigate much larger porphyrins with interesting optical properties.

\section{A. Benzene}

The electronic ground state of benzene is a closed shell well described by a single configuration. It is, therefore, well adapted to calculation of singlet excitation energies using a coupled cluster linear response function. Our goal is to verify the quality of the results and, therefore, the error introduced by the Cholesky decomposition as well as the computational cost of the method. All calculations were done at the same geometry from Wannere and Schleyer. ${ }^{28}$ We have carried out a set of calculations using three different implementations of the CC2 linear response. The first one employs a standard Davidson algorithm for the diagonalization of the full CC2 Jacobian matrix (Eq. (8)) without using the Cholesky decomposition. The convergence criterion for the Davidson algorithm is fixed to $10^{-3}$. We will refer to this implementation as full-CC2. In the second implementation the reduced effective Jacobian CC2 matrix is diagonalized using Cholesky vectors for the decomposition of the two-electron integrals. For this implementation a standard Davidson algorithm is used until self-consistency. The criterion for the Davidson part is also fixed to $10^{-3}$ while the self-consistency parameter is chosen to be $10^{-4}$. This implementation will be denoted CD-CC2std. The last method is equivalent to the second one with an optimized Davidson algorithm as described in Sec. III B with a criterion of $10^{-4}$ for self-consistency. Because the Davidson threshold is given by $\tau=\left|\omega_{\text {input }}-\omega^{j}\right|$, excitation energies are further converged compared to the previous algorithms.

For each of those three implementation we performed calculations using the hierarchy of correlation consistent basis sets of Dunning, ${ }^{29}$ aug-cc-pVXZ for $\mathrm{X}:=\{\mathrm{D}, \mathrm{T}, \mathrm{Q}, 5\}$. In the case of the full-CC2 algorithm, the quintuple- $\zeta$ basis set was not tractable due to the very high computational cost. For the Cholesky decomposition, we performed calculation using three different thresholds, $\tau_{1}=10^{-8}, \tau_{2}=10^{-6}$, and $\tau_{3}=10^{-4}$. All excitation energy calculations were done using a local version of the DALTON program in which the above algorithms have been implemented. ${ }^{30}$ The results for the lowest singlet excited states of benzene are shown in Table II.

The results presented in Table II show no significant deviations with or without using the Cholesky decomposition. Differences appear around the third or fourth decimal and come from the fact that different optimization processes were employed. However, the CC2 excitation energies are known

TABLE II. Vertical excitation energies (in eV) for the lowest singlet excited states of benzene obtained with the CC2 model.

\begin{tabular}{|c|c|c|c|c|c|c|c|c|}
\hline \multirow{2}{*}{$\begin{array}{l}\text { One electron basis: } \\
\text { Cholesky parameter: }\end{array}$} & \multicolumn{4}{|c|}{ aug-cc-pVDZ } & \multicolumn{4}{|c|}{ aug-cc-pVTZ } \\
\hline & $(0)^{\mathrm{a}}$ & $\left(10^{-8}\right)^{\mathrm{b}}$ & $\left(10^{-6}\right)^{\mathrm{c}}$ & $\left(10^{-4}\right)^{\mathrm{c}}$ & $(0)^{\mathrm{a}}$ & $\left(10^{-8}\right)^{\mathrm{b}}$ & $\left(10^{-6}\right)^{\mathrm{c}}$ & $\left(10^{-4}\right)^{\mathrm{c}}$ \\
\hline \# Cholesky vectors: & $\ldots$ & 18528 & 1536 & 929 & $\ldots$ & 85905 & 3135 & 1932 \\
\hline $1^{1} A_{1 u}$ & 7.036 & 7.036 & 7.036 & 7.036 & 7.152 & 7.152 & 7.152 & 7.152 \\
\hline $1^{1} A_{2 u}$ & 6.885 & 6.885 & 6.885 & 6.885 & 7.006 & 7.006 & 7.006 & 7.006 \\
\hline $1^{1} B_{1 g}$ & 7.567 & 7.567 & 7.567 & 7.567 & 7.668 & 7.668 & 7.668 & 7.668 \\
\hline $1^{1} B_{1 u}$ & 6.506 & 6.506 & 6.505 & 6.505 & 6.466 & $\ldots{ }^{\mathrm{d}}$ & 6.466 & 6.466 \\
\hline $1^{1} B_{2 u}$ & 5.279 & 5.279 & 5.279 & 5.279 & 5.237 & 5.237 & 5.237 & 5.237 \\
\hline $1^{1} E_{1 g}$ & 6.352 & 6.352 & 6.352 & 6.352 & 6.475 & 6.475 & 6.475 & 6.475 \\
\hline $2{ }^{1} E_{1 g}$ & 7.577 & 7.577 & 7.579 & 7.577 & 7.676 & 7.676 & 7.676 & 7.676 \\
\hline $1^{1} E_{1 u}$ & 7.177 & 7.177 & 7.177 & 7.177 & 7.143 & 7.143 & 7.143 & 7.143 \\
\hline $1^{1} E_{2 g}$ & 8.741 & 8.741 & 8.741 & 8.741 & 8.512 & 8.511 & 8.511 & 8.512 \\
\hline $2{ }^{1} E_{2 g}$ & 8.829 & 8.829 & 8.829 & 8.829 & 8.596 & 8.595 & 8.595 & 8.596 \\
\hline $1^{1} E_{2 u}$ & 6.949 & 6.949 & 6.949 & 6.949 & 7.068 & 7.068 & 7.068 & 7.068 \\
\hline One electron basis: & \multicolumn{4}{|c|}{ aug-cc-pVQZ } & \multicolumn{4}{|c|}{ aug-cc-pV5Z } \\
\hline Cholesky parameter: & $(0)^{\mathrm{a}}$ & $\left(10^{-8}\right)^{\mathrm{b}}$ & $\left(10^{-6}\right)^{\mathrm{c}}$ & $\left(10^{-4}\right)^{\mathrm{c}}$ & $(0)^{\mathrm{a}}$ & $\left(10^{-8}\right)^{\mathrm{b}}$ & $\left(10^{-6}\right)^{\mathrm{c}}$ & $\left(10^{-4}\right)^{\mathrm{c}}$ \\
\hline \# Cholesky vectors: & $\ldots$ & 286146 & 5358 & 3479 & $\ldots$ & 771903 & 8645 & 5878 \\
\hline $1^{1} A_{1 u}$ & 7.196 & 7.196 & 7.196 & 7.196 & $\ldots$ & 7.207 & 7.207 & 7.207 \\
\hline $1^{1} A_{2 u}$ & 7.053 & 7.053 & 7.053 & 7.053 & $\ldots$ & 7.067 & 7.067 & 7.067 \\
\hline $1^{1} B_{1 g}$ & 7.707 & 7.707 & 7.707 & 7.707 & $\ldots$ & 7.708 & 7.708 & 7.707 \\
\hline $1^{1} B_{1 u}$ & 6.461 & $\ldots{ }^{d}$ & 6.461 & 6.461 & $\ldots$ & $\ldots{ }^{d}$ & 6.459 & 6.459 \\
\hline $1^{1} B_{2 u}$ & 5.227 & 5.227 & 5.227 & 5.227 & $\ldots$ & 5.224 & 5.224 & 5.224 \\
\hline $1^{1} E_{1 g}$ & 6.525 & 6.525 & 6.525 & 6.525 & $\ldots$ & 6.543 & 6.543 & 6.544 \\
\hline $2{ }^{1} E_{1 g}$ & 7.714 & 7.714 & 7.714 & 7.707 & $\ldots$ & 7.720 & 7.720 & 7.708 \\
\hline $1^{1} E_{1 u}$ & 7.136 & 7.136 & 7.136 & 7.136 & $\ldots$ & 7.127 & 7.127 & 7.127 \\
\hline $1^{1} E_{2 g}$ & 8.345 & 8.345 & 8.345 & 8.345 & $\ldots$ & 8.231 & 8.231 & 8.232 \\
\hline $2{ }^{1} E_{2 g}$ & 8.399 & 8.399 & 8.399 & 8.399 & $\ldots$ & 8.272 & 8.272 & 8.272 \\
\hline $1^{1} E_{2 u}$ & 7.114 & 7.114 & 7.114 & 7.114 & $\ldots$ & 7.127 & 7.127 & 7.127 \\
\hline
\end{tabular}

${ }^{\mathrm{a}}$ Full-CC2.

${ }^{\mathrm{b}} \mathrm{CD}-\mathrm{CC} 2$-std.

${ }^{\mathrm{c}} \mathrm{CD}$-CC2-opt.

${ }^{\mathrm{d}}$ Not converged. 


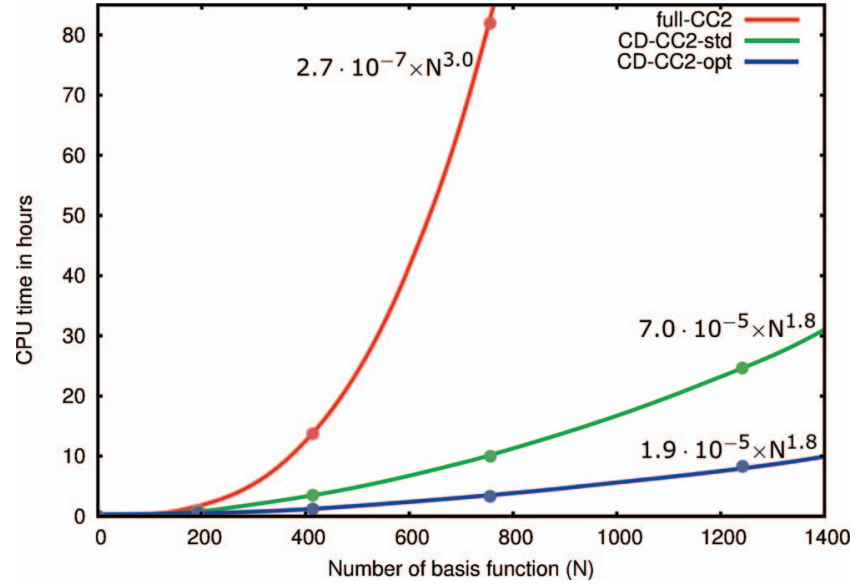

FIG. 1. Comparison of the scaling of the different algorithms with respect to basis set size for the CC2 excitation energy calculations on the benzene molecule.

to show errors of the order of $0.2-0.3 \mathrm{eV}^{15,16}$ and, therefore, neither the Cholesky decomposition nor the differences in the optimization process impact the quality of the excitation energies. We note that the CD-CC2-opt algorithm not only makes the calculation faster, but also helps convergence in some cases, see, for instance, the lowest state of symmetry ${ }^{1} B_{1 u}$ in Table II.

In order the Cholesky decomposition to be useful, the number of Cholesky vectors $K$ must be much lower than the number of distinct elements of the product space of the onecenter basis functions, $(K \ll N(N+1) / 2) .{ }^{18}$ In our calculations, this condition was always widely fulfilled. Obviously, the calculations are faster and less accurate when the number of Cholesky vectors decreases. In practice, a parameter of $10^{-4}-10^{-6}$ has proven to be enough. ${ }^{31}$

In Figure 1, we have plotted the time in hours corresponding to the coupled cluster part of the calculations against the number of basis functions for the computation of the lowest singlet excited states of benzene. For each algorithm, we performed a power fit of the form $a \times N^{b}$ with $N$ the number of basis functions. It shows that using the Cholesky decomposition considerably reduces the power in the scaling of the methods (parameter $b$ ) while using the optimized Davidson algorithm impacts the pre-factor $a$. The improvement due to $\mathrm{CD}$ is quite impressive and undeniably expand the applicability of the $\mathrm{CC} 2$ linear response scheme to calculation of excitation energies of bigger system with larger basis set.

In order to further analyze the capacities of the Cholesky decomposition in the calculation of excitation energies, we looked at the computational cost for the construction of one linear transformed vector. For the small basis set aug-ccpVDZ, the difference between full-CC2 and CD-CC2 with a parameter of $10^{-6}$ is negligible. The standard CC2 is even a little faster in average due to the fact that the Cholesky implementation involves more I/O operations that become valuable only for bigger basis set. Indeed, when dealing with the aug-cc-pVQZ basis set, the CD-CC2 transformation is almost 7 times faster than the non-direct full-CC2 algorithm, showing again the efficiency of the Cholesky decomposition.
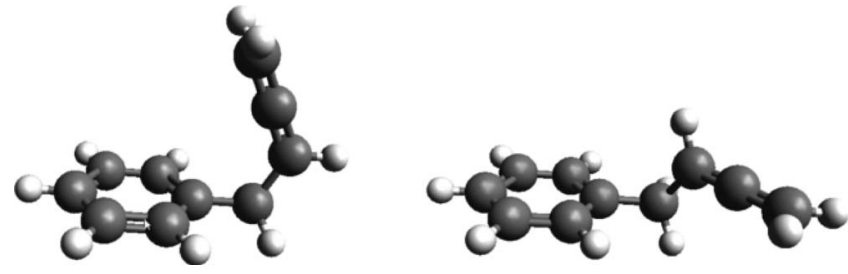

FIG. 2. Structures of benzylallene conformers, gauche (left) and anti (right).

\section{B. Benzylallene}

Benzylallene is the minor product of the recombination of benzyl and propargyl radicals. Because of the flexibility in the carbon chain, there are two possible isomers, one with the chain pointed away from the benzene (anti) and the other with the side-chain over the benzene ring (gauche), see Figure 2. Since there is a chance that this molecule acts as photochemical precursor in the formation of bicyclic rings-for instance in the atmosphere of Titan-it is important to study the spectroscopic behavior of both isomers. ${ }^{32}$

We chose the benzylallene conformers to perform a second test of our implementation. No exploitation of symmetry point group can be done and calculation of excitation energies can rapidly become intractable. It is important to remind that in order to be reliable, the excitation energies need to be treated with relatively large basis set. A Cholesky decomposition algorithm is therefore well adapted for the treatment of this system. Moreover, as for benzene, the ground state of the systems are single-configurational closed shell.

In order to properly treat the excited states, we first performed a rough calculation of the 15 lowest singlet excited states using the poor Sadlej-pVTZ basis set. The same calculation was repeated by adding diffuse basis function at the center of mass of the system as proposed by Kaufmann et al. in Ref. 33 in order to stabilize the Rydberg states. These steps allow us to localize and remove the Rydberg states in order to focus ourselves in the description of the two lowest valence singlet states.

We decided to perform an extrapolation to the $\mathrm{CBS}^{34,35}$ of the two lowest singlet states of both conformers as they seem to play important spectroscopic roles. ${ }^{32}$ For this purpose, we used the hierarchy of correlation consistent basis set of Dunning, aug-cc-pVXZ with $\mathrm{X}:=\{\mathrm{D}, \mathrm{T}, \mathrm{Q}\} .^{29} \mathrm{Ge}-$ ometries of both isomers were optimized at the B3LYP/6$311 \mathrm{G} * *$ level $^{36,37}$ using GAUSSIAN09. ${ }^{38}$ The extrapolation to complete basis was done by independent calculations of the non-correlated, $S_{i, \mathrm{CBS}}^{\mathrm{CCS}}$ and the correlated, $S_{i, \mathrm{CBS}}^{\mathrm{corr}}$ contributions,

TABLE III. Vertical excitation energies (in eV) for the two lowest valence singlet states of conformer anti of benzylallene.

\begin{tabular}{lllllll}
\hline \hline State & $S_{1}^{\mathrm{CCS}}$ & $S_{2}^{\mathrm{CCS}}$ & $S_{1}^{\mathrm{corr}}$ & $S_{2}^{\mathrm{corr}}$ & $S_{1}^{\mathrm{CC} 2}$ & $S_{2}^{\mathrm{CC} 2}$ \\
\hline$X=\mathrm{D}$ & 5.943 & 6.061 & -0.768 & -0.088 & 5.176 & 5.974 \\
$X=\mathrm{T}$ & 5.937 & 6.047 & -0.799 & -0.085 & 5.138 & 5.962 \\
$X=\mathrm{Q}$ & 5.935 & 6.045 & -0.805 & -0.081 & 5.129 & 5.964 \\
$\mathrm{CBS}$ & 5.934 & 6.045 & -0.810 & -0.079 & 5.124 & 5.967 \\
\hline \hline
\end{tabular}


TABLE IV. Vertical excitation energies (in eV) for the two lowest valence singlet states of conformer gauche of benzylallene.

\begin{tabular}{lllllll}
\hline \hline State & $S_{1}^{\mathrm{CCS}}$ & $S_{2}^{\mathrm{CCS}}$ & $S_{1}^{\mathrm{corr}}$ & $S_{2}^{\mathrm{corr}}$ & $S_{1}^{\mathrm{CC} 2}$ & $S_{2}^{\mathrm{CC} 2}$ \\
\hline$X=\mathrm{D}$ & 5.963 & 6.104 & -0.770 & -0.175 & 5.193 & 5.929 \\
$X=\mathrm{T}$ & 5.956 & 6.087 & -0.802 & -0.153 & 5.154 & 5.934 \\
$X=\mathrm{Q}$ & 5.954 & 6.085 & -0.809 & -0.144 & 5.145 & 5.942 \\
$\mathrm{CBS}$ & 5.954 & 6.085 & -0.814 & -0.137 & 5.140 & 5.948 \\
\hline \hline
\end{tabular}

according to

$$
\begin{gathered}
S_{i, \mathrm{CBS}}=S_{i, \mathrm{CBS}}^{\mathrm{CCS}}+S_{i, \mathrm{CBS}}^{\mathrm{corr}}, \\
S_{i, \mathrm{CBS}}^{\mathrm{CCS}}=\lim _{X \rightarrow \infty}\left(a+b e^{-c X}\right), \\
S_{i, \mathrm{CBS}}^{\mathrm{corr}}=\frac{S_{i, X}^{\mathrm{corr}} X^{3}-S_{i, X-1}^{\mathrm{corr}}(X-1)^{3}}{X^{3}-(X-1)^{3}},
\end{gathered}
$$

where $i=1,2$, corresponds to the two lowest excited states and $X$ is the cardinal of the corresponding aug-cc-pVXZ basis. The non-correlated excitation energies are calculated, also using Cholesky decomposed two-electron integrals, at the CCS level which is equivalent to CI Singles (CIS). In Tables III and IV we have listed excitation energies of the two lowest singlet states for both isomers.

We noticed in Tables III and IV that CCS excitation energies are almost converged to CBS using the aug-cc-pVQZ basis set whereas the $\mathrm{CC} 2$ results converged much slower. These results therefore justify the utilization of large basis set and CBS extrapolation for excitation energy calculations.

The previous basis set investigation on the benzylallene isomers shows that the Cholesky decomposition of the twoelectron integrals is a really powerful tool, confirming again its capacity to reduce the computational cost and memory requirements in electronic structure calculations.
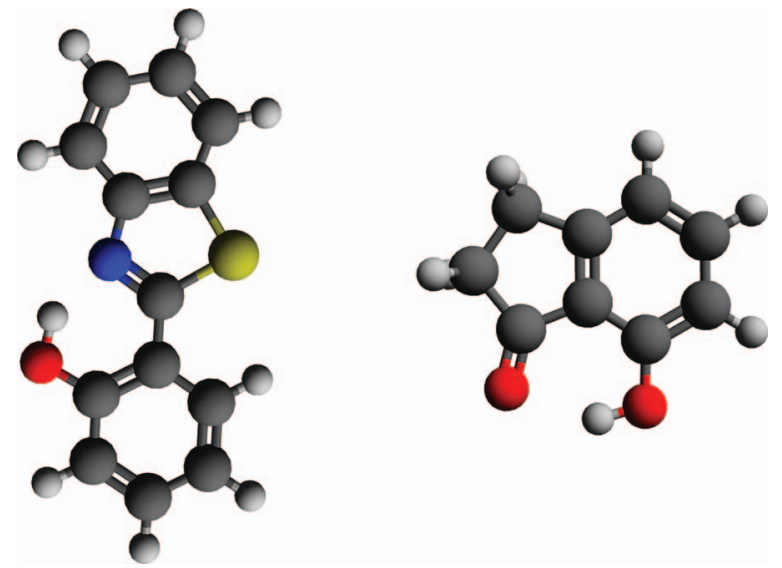

FIG. 3. Structures of HBT (left) and 7HIN (right) molecular systems.

\section{7-Hydroxy-1-indanone and 2-(2'-hydroxy-phenyl)benzothiazole}

7-Hydroxy-1-indanone (7HIN) and 2-(2'-hydroxyphenyl)benzothiazole (HBT) (see Fig. 3) are examples of molecular systems presenting excited-state intramolecular proton transfer (ESIPT), a process of great interest in photochemistry and photobiology and which has technological implications in laser-dyes or UV filters. Furthermore, both 7HIN and HBT have been used to test the reliability of ESIPT potential energy surfaces calculated with the RI-CC2 approach among other methods. ${ }^{39}$ Here, we use the $\mathrm{CC} 2$ geometry for the ground state optimized by Aquino et al. ${ }^{39}$ and calculate the excitation energies to the states $2^{1} \mathrm{~A}^{\prime}$ and $1^{1} \mathrm{~A}^{\prime \prime}$ corresponding, respectively, to the $\pi \rightarrow \pi^{*}$ and $n \rightarrow \pi^{*}$ transitions.

It has been found that because of the systematic character of the error due to $\mathrm{CD}$, the $\mathrm{CD}$ approximation leads essentially to a uniform shift of the whole spectrum of ionization potentials. ${ }^{40}$ This suggests the possibility of reducing the computational cost of the determination of $\mathrm{CC} 2$ excitation

TABLE V. Comparison of the CC2/TZVP accuracy of excitation energies of 7HIN (eV) for different Cholesky thresholds.

\begin{tabular}{lccccc}
\hline \hline Thresholds $^{\mathrm{a}}$ & $\operatorname{dim}(\mathrm{HF})^{\mathrm{b}}$ & $\operatorname{dim}(\mathrm{CC} 2)^{\mathrm{c}}$ & HF energy $\left(E_{h}\right)$ & CC2 energy $\left(E_{h}\right)$ & $\pi \rightarrow \pi^{*}$ \\
\hline Standard & 46359 & 6104 & -495.36921610 & -497.0218072 & 4.288 \\
RI-CC2 $^{\mathrm{d}}$ & $\ldots \mathrm{e}^{\mathrm{e}}$ & 692 & -495.36921610 & -497.021442 & 4.29 \\
$10^{-8}-10^{-8}$ & 3352 & 3352 & -495.36921607 & -497.0218071 & 4.111 \\
$10^{-8}-10^{-6}$ & 3352 & 2011 & -495.36921607 & -497.0217957 & 4.288 \\
$10^{-8}-10^{-4}$ & 3352 & 1131 & -495.36921607 & -497.0217692 & 4.288 \\
$10^{-6}-10^{-6}$ & 2011 & 2011 & -495.36921321 & -497.0217926 & 4.289 \\
$10^{-6}-10^{-4}$ & 2011 & 1131 & -495.36921321 & -497.0204471 & 4.288 \\
$10^{-8}-10^{-8}$ & 3352 & $1860^{\mathrm{f}}$ & -495.36921607 & -497.0218071 & 4.111 \\
$10^{-8}-10^{-6}$ & 3352 & $1091^{\mathrm{f}}$ & -495.36921607 & -497.0217957 & 4.288 \\
$10^{-8}-10^{-4}$ & 3352 & $597^{\mathrm{f}}$ & -495.36921607 & -497.0204435 & 4.289 \\
$10^{-6}-10^{-6}$ & 2011 & $1088^{\mathrm{f}}$ & -495.36921321 & -497.0217926 & 4.288 \\
$10^{-6}-10^{-4}$ & 2011 & $597^{\mathrm{f}}$ & -495.36921321 & -497.0204471 & 4.109 \\
\hline \hline
\end{tabular}

a For $\mathrm{HF}$ and $\mathrm{CC} 2$, respectively.

${ }^{\mathrm{b}}$ Number of Cholesky vectors to represent $(\alpha \beta \mid \gamma \delta)$.

${ }^{\mathrm{c}}$ Number of Cholesky vectors to represent $(a i \hat{\mid} b j)$.

${ }^{\mathrm{d}}$ Reference 39 .

${ }^{\mathrm{e}}$ Standard HF calculation.

${ }^{\mathrm{f}}$ Using decomposed $(a i \hat{\mid} b j)$ integrals. 
TABLE VI. Comparison of the CC2/TZVP accuracy of excitation energies of HBT (eV) for different Cholesky thresholds.

\begin{tabular}{lccccc}
\hline \hline Thresholds $^{\mathrm{a}}$ & $\operatorname{dim}(\mathrm{HF})^{\mathrm{b}}$ & $\operatorname{dim}(\mathrm{CC} 2)^{\mathrm{c}}$ & HF energy $\left(E_{h}\right)$ & CC2 energy $\left(E_{h}\right)$ & $\pi \rightarrow \pi^{*}$ \\
\hline Standard & 65341 & 12986 & -1024.57103801 & -1026.9075110 & 3.935 \\
RI-CC2 ${ }^{\mathrm{d}}$ & $\ldots \mathrm{e}^{-8}$ & 989 & -1024.57103801 & -1026.877375 & 3.94 \\
$10^{-8}-10^{-6}$ & 4631 & 4631 & -1024.57103795 & -1026.9075109 & 3.935 \\
$10^{-8}-10^{-6}$ & 4631 & 2750 & -1024.57103795 & -1026.9075075 & 3.935 \\
$10^{-8}-10^{-4}$ & 4631 & 1580 & -1024.57103795 & -1026.9075741 & 3.934 \\
$10^{-6}-10^{-6}$ & 2750 & 2750 & -1024.57103296 & -1026.9075027 & 3.935 \\
$10^{-6}-10^{-4}$ & 2750 & 1580 & -1024.57103296 & -1026.9075679 & 3.303 \\
$10^{-8}-10^{-8}$ & 4631 & $2580^{\mathrm{f}}$ & -1024.57103795 & -1026.9075107 & 5.303 \\
$10^{-8}-10^{-6}$ & 4631 & $1511^{\mathrm{f}}$ & -1024.57103795 & -1026.9074848 & 3.935 \\
$10^{-8}-10^{-4}$ & 4631 & $819^{\mathrm{f}}$ & -1024.57103795 & -1026.9033140 & 3.935 \\
$10^{-6}-10^{-6}$ & 2750 & $1510^{\mathrm{f}}$ & -1024.57103296 & -1026.9074809 & 3.933 \\
$10^{-6}-10^{-4}$ & 2750 & $816^{\mathrm{f}}$ & -1024.57103296 & -1026.9033516 & 3.935 \\
\hline \hline
\end{tabular}

${ }^{\mathrm{a}}$ For $\mathrm{HF}$ and $\mathrm{CC} 2$, respectively.

${ }^{\mathrm{b}}$ Number of Cholesky vectors to represent $(\alpha \beta \mid \gamma \delta)$.

${ }^{\mathrm{c}}$ Number of Cholesky vectors to represent $(a i \hat{\mid} b j)$.

${ }^{\mathrm{d}}$ Reference 39 .

eStandard HF calculation.

${ }^{\mathrm{f}}$ Using decomposed $(a i \hat{\imath} b j)$ integrals.

energies by using dual threshold calculations. So, we have explored whether it is advantageous to use a higher threshold for CC2 than for HF. The results presented in Tables V and VI show that this is only in part the case. Indeed, a rather loose threshold of $\tau=10^{-4}$ is enough to get excitation energies with errors of no more than a couple of $\mathrm{meV}$, while the error in the absolute energy of the ground state is sensibly larger. However, at the same time no significant improvement of the excitation energies is obtained by using a tighter threshold for the underlying Hartree-Fock calculation.

On the other hand, an improvement of the computational cost may be obtained by explicitly decomposing the integrals $(a i \hat{\mid} b j)$. This effect can be checked by comparing the number of vectors used to represent those integrals depending on whether they are or are not decomposed (see Tables V and VI). In the latter case, the $\mathrm{AO}\left\{L_{\alpha \beta}^{J}\right\}$ Cholesky basis was simply transformed to MO basis with the hole and particle $\Lambda$ matrices (so keeping the number of vectors), while in the former the MO $\left\{\hat{L}_{a i}^{J}\right\}$ basis was directly constructed by decomposing the $(a i \hat{\imath} \mid b j)$ integrals calculated on-the-fly. Moreover, we stress the fact that even using the loosest threshold the Cholesky decomposition-based algorithm provides results of at least the same accuracy than RI-based methods, with the $\mathrm{CD}$ inherent advantage of easy error control.

TABLE VII. Comparison of DIIS extrapolation and Davidson algorithm to solve the pseudo-eigenvalue problem.

\begin{tabular}{lcc}
\hline \hline & DIIS extrapolation & Davidson algorithm \\
\hline$A^{\prime} \Delta E(\mathrm{eV})$ & $3.935,4.449,4.828$ & $3.935,4.449,4.828$ \\
$A^{\prime} \# \mathrm{LT}^{\mathrm{a}}$ & 52 & 71 \\
$A^{\prime \prime} \Delta E(\mathrm{eV})$ & $5.303,5.714,5.992$ & $5.303,5.714,5.992$ \\
$A^{\prime \prime} \# \mathrm{LT}^{\mathrm{b}}$ & 65 & 88 \\
Saving $^{\mathrm{c}}$ & $88.84 \%$ & $85.41 \%$ \\
\hline \hline
\end{tabular}

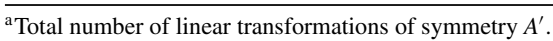

b Total number of linear transformations of symmetry $A^{\prime \prime}$.

${ }^{\mathrm{c}}$ Relative to full-CC2.
To finish this subsection, in Table VII a comparison of the two different ways discussed in Sec. III B to solve the pseudoeigenvalue problem is presented. Of course, the computed excitation energies are exactly the same until the required convergence threshold. As expected and previously mentioned, DIIS extrapolation speeds up the process, as reflected by the fact that approximately one-third more of linear transformations are required by the modified Davidson algorithm. At any rate, when comparing the Davidson algorithm to standard $\mathrm{CC} 2$, the saving is still very large, although of course smaller than that of the DIIS implementation. Finally, it is worthy to recall that the use of Davidson algorithm as preconditioner in the DIIS extrapolation is unavoidable to prevent convergence problems.

\section{Free base porphin}

Because of their interesting properties and their role in biological systems, porphyrins have been extensively studied in the literature. Their optical properties have been of particular interest, for example, in the development of dye-sensitized solar cells, ${ }^{41}$ and many theoretical studies have been trying

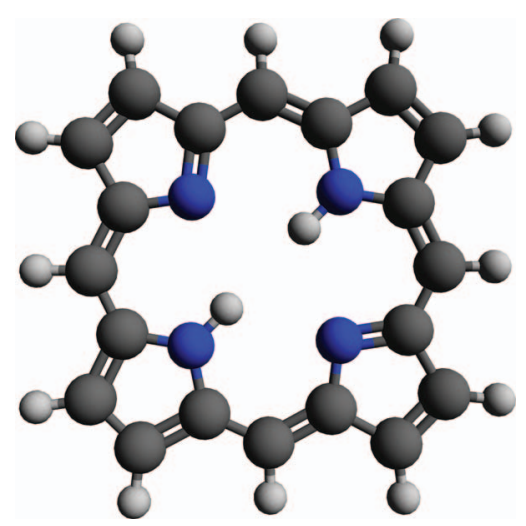

FIG. 4. Structure of the free base porphin ( $D_{2 h}$ symmetry) 
TABLE VIII. Vertical excitation energies (in eV) for the lowest singlet excited states of free base porphin obtained with the CC2 model.

\begin{tabular}{lccc}
\hline \hline Cholesky parameter: & $(0)^{\mathrm{a}}$ & $\left(10^{-6}\right)^{\mathrm{b}}$ & $\left(10^{-4}\right)^{\mathrm{b}}$ \\
\hline $1^{1} A_{g}$ & 3.998 & 3.998 & 3.999 \\
$2^{1} A_{g}$ & 4.278 & 4.278 & 4.276 \\
$3^{1} A_{g}$ & 4.712 & 4.712 & 4.713 \\
$1^{1} B_{3 u}$ & 4.337 & 4.337 & 4.338 \\
$2^{1} B_{3 u}$ & 6.433 & 6.434 & 6.434 \\
$3^{1} B_{3 u}$ & 7.009 & 7.009 & 7.009 \\
$1^{1} B_{2 u}$ & 2.683 & 2.683 & 2.683 \\
$2^{1} B_{2 u}$ & 3.581 & 3.581 & 3.580 \\
$3^{1} B_{2 u}$ & 4.213 & 4.213 & 4.214 \\
$1^{1} B_{1 g}$ & 4.318 & 4.319 & 4.319 \\
$2^{1} B_{1 g}$ & 6.792 & 6.792 & 6.792 \\
$3^{1} B_{1 g}$ & 6.998 & 6.998 & 6.996 \\
$1^{1} B_{1 u}$ & 2.304 & 2.304 & 2.303 \\
$2^{1} B_{1 u}$ & 3.511 & 3.511 & 3.510 \\
$3^{1} B_{1 u}$ & 4.182 & 4.182 & 4.183 \\
$1^{1} B_{2 g}$ & 4.120 & 4.120 & 4.120 \\
$2^{1} B_{2 g}$ & 6.132 & 6.132 & 6.132 \\
$3^{1} B_{2 g}$ & 6.727 & 6.727 & 6.727 \\
$1^{1} B_{3 g}$ & 3.774 & 3.775 & 3.775 \\
$2^{1} B_{3 g}$ & 4.133 & 4.133 & 4.133 \\
$3^{1} B_{3 g}$ & 4.545 & 4.545 & 4.545 \\
$1^{1} A_{u}$ & 4.124 & 4.124 & 4.124 \\
$2^{1} A_{u}$ & 6.117 & 6.117 & 6.116 \\
$3^{1} A_{u}$ & 6.687 & 6.687 & 6.686 \\
\hline \hline
\end{tabular}

${ }^{\mathrm{a}}$ Full-CC2.

${ }^{\mathrm{b}} \mathrm{CD}-\mathrm{CC} 2$-opt.

to describe the electronic excitation spectrum of free base porphin. ${ }^{42-44}$ In this section, we present another test of our implementation on the free base porphin (see Fig. 4).

The symmetry of the molecule $\left(\mathrm{D}_{2 h}\right)$ allows us to perform cheap calculations using different implementation of the CC2 linear response theory. This study could thus be used as a starting point for the investigation of larger porphyrins for which a full-CC2 calculation is not affordable. We proceeded with the same strategy as for benzene in Sec. IV A. A total of 24 singlet excitation energies have been calculating using the TZVP basis set ${ }^{45}$ and two different algorithms, full-CC2 and CD-CC2-opt. The Cholesky calculations were performed with two different thresholds, $\tau_{1}=10^{-6}$ and $\tau_{2}=10^{-4}$.

The results presented in Table VIII show the same behavior as for the benzene molecules, i.e., the $\mathrm{CD}-\mathrm{CC} 2$ results are basically the same as the one with the full-CC2 algorithm. The Cholesky algorithm could thus be used to treat larger and more complex porphyrins.

\section{CONCLUSIONS}

The goal of this work was to implement and to test a new method for the calculation of accurate vertical excitation energies applicable to larger systems and larger basis sets.

For this purpose, we chose to reduce the scaling of the CC2 linear response scheme using a Cholesky decomposition of the two-electron integral matrix. We showed in Sec. III how such a method can be efficiently implemented. In Sec. IV, we performed a set of calculations that confirm both the quality of the excitation energies and the reduction of the computational cost. The obtained results prove the ability of Cholesky decomposition based techniques to reproduce standard CC2 excitation energies at a much lower computational cost and with no relevant loss of accuracy.

\section{ACKNOWLEDGMENTS}

Pablo Baudin acknowledges support from the Erasmus Mundus program of the European Union. Economical funding from the Spanish Ministerio de Economía y Competividad project CTQ2010-19738 is also acknowledged.

${ }^{1}$ P. Pulay and S. Sæbo, Theor. Chim. Acta 69, 357 (1986).

${ }^{2}$ S. Sæbo and P. Pulay, J. Chem. Phys. 86, 914 (1987).

${ }^{3}$ M. Schütz, G. Hetzer, and H.-J. Werner, J. Chem. Phys. 111, 5691 (1999).

${ }^{4}$ I.-M. Høyvik, K. Kristensen, B. Jansík, and P. Jørgensen, J. Chem. Phys. 136, 014105 (2012).

${ }^{5}$ F. Aquilante, R. Lindh, and T. B. Pedersen, J. Chem. Phys. 127, 114107 (2007).

${ }^{6}$ F. Weigend, M. Kattannek, and R. Ahlrichs, J. Chem. Phys. 130, 164106 (2009).

${ }^{7}$ V. Khoromskaia, B. Khoromskij, and R. Schneider, SIAM J. Comput. 35, A987 (2013).

${ }^{8}$ C. L. Janssen and I. M. B. Nielsen, Parallel Computing in Quantum Chemistry (CRC Press, Boca Raton, 2008).

${ }^{9}$ M. Valiev, E. Bylaska, N. Govind, K. Kowalski, T. Straatsma, H. V. Dam, D. Wang, J. Nieplocha, E. Apra, T. Windus, and W. de Jong, Comput. Phys. Commun. 181, 1477 (2010).

${ }^{10}$ J. E. Stone, D. J. Hardy, I. S. Ufimtsev, and K. Schulten, J. Mol. Graph. Model. 29, 116 (2010).

${ }^{11}$ A. W. Götz, T. Wölfle, and R. C. Walker, in Annual Reports in Computational Chemistry, edited by R. A. Wheeler (Elsevier, 2010), Vol. 6, pp. 21-35.

${ }^{12}$ K. Morokuma, Philos. Trans. R. Soc. London 360, 1149 (2002)

${ }^{13}$ T. B. Pedersen, A. Sánchez de Merás, and H. Koch, J. Chem. Phys. 120, 8887 (2004).

${ }^{14}$ O. Christiansen, H. Koch, and P. Jørgensen, Chem. Phys. Lett. 243, 409 (1995).

${ }^{15}$ O. Christiansen, H. Koch, P. Jørgensen, and J. Olsen, Chem. Phys. Lett. 256, 185 (1996).

${ }^{16}$ H. Koch, O. Christiansen, P. Jørgensen, and J. Olsen, Chem. Phys. Lett. 244, 75 (1995).

${ }^{17}$ F. Aquilante, L. Boman, J. Boström, H. Koch, R. Lindh, A. Sánchez de Merás, and T. B. Pedersen, in Linear Scaling Techniques in Computational Chemistry and Physics, Challenges and Advances in Computational Chemistry and Physics Vol. 13, edited by R. Zalesny, M. G. Papadopoulos, P. G. Mezey, and J. Leszczynski (Springer, Netherlands, 2011), Chap. 13, pp. 301-343.

${ }^{18}$ H. Koch, A. Sánchez de Merás, and T. B. Pedersen, J. Chem. Phys. 118, 9481 (2003).

${ }^{19}$ H. Koch, O. Christiansen, P. Jørgensen, A. Sánchez de Merás, and T. Helgaker, J. Chem. Phys. 106, 1808 (1997).

${ }^{20} \mathrm{O}$. Christiansen, A. Halkier, H. Koch, P. Jørgensen, and T. Helgaker, J. Chem. Phys. 108, 2801 (1998).

${ }^{21}$ C. Hättig and F. Weigend, J. Chem. Phys. 113, 5154 (2000).

${ }^{22}$ C. Hättig, A. Kohn, and K. Hald, J. Chem. Phys. 116, 5401 (2002).

${ }^{23}$ C. Hättig and A. Kohn, J. Chem. Phys. 117, 6939 (2002).

${ }^{24}$ C. Hättig, J. Chem. Phys. 118, 7751 (2003).

${ }^{25}$ T. B. Pedersen and H. Koch, J. Chem. Phys. 106, 8059 (1997).

${ }^{26}$ T. Helgaker, S. Coriani, P. Jørgensen, K. Kristensen, J. Olsen, and K. Ruud, Chem. Rev. 112, 543 (2012).

${ }^{27}$ M. Crouzeix, B. Philippe, and M. Sadkane, SIAM J. Comput. 15, 62 (1994).

${ }^{28}$ C. S. Wannere and P. v. R. Schleyer, Org. Lett. 5, 865 (2003).

${ }^{29}$ T. H. Dunning, Jr., J. Chem. Phys. 90, 1007 (1989).

${ }^{30}$ DALTON, a molecular electronic structure program, Release Dalton2011 (2011), see http://daltonprogram.org/.

${ }^{31}$ J. Boström, M. G. Delcey, F. Aquilante, L. Serrano-Andrés, T. B. Pedersen, and R. Lindh, J. Chem. Theory Comput. 6, 747 (2010). 
${ }^{32}$ J. A. Sebree, N. M. Kidwell, T. M. Selby, B. K. Amberger, R. J. McMahon, and T. S. Zwier, J. Am. Chem. Soc. 134, 1153 (2012).

${ }^{33}$ K. Kaufmann, W. Baumeister, and M. Jungen, J. Phys. B 22, 2223 (1989).

${ }^{34}$ D. Feller, J. Chem. Phys. 96, 6104 (1992).

${ }^{35}$ T. Helgaker, W. Klopper, H. Koch, and J. Noga, J. Chem. Phys. 106, 9639 (1997).

${ }^{36}$ A. D. Becke, Phys. Rev. A 38, 3098 (1988).

${ }^{37}$ C. Lee, W. Yang, and R. G. Parr, Phys. Rev. B 37, 785 (1988)

${ }^{38}$ M. J. Frisch, G. W. Trucks, H. B. Schlegel et al., GAussian 09, Revision A.1, Gaussian, Inc., Wallingford, CT, 2009.
${ }^{39}$ A. Aquino, H. Lischka, and C. Hättig, J. Phys. Chem. A 109, 3201 (2005).

${ }^{40}$ V. Vysotskiy and L. Cederbaum, J. Chem. Phys. 132, 044110 (2010).

${ }^{41}$ M. G. Walter, A. B. Rudine, and C. C. Wamser, J. Porphyrins Phthalocyanines 14, 759 (2010).

${ }^{42}$ M. Merchán, E. Ortí, and B. O. Roos, Chem. Phys. Lett. 226, 27 (1994).

${ }^{43}$ S. R. Gwaltney and R. J. Bartlett, J. Chem. Phys. 108, 6790 (1998).

${ }^{44}$ A. Kerridge, Phys. Chem. Chem. Phys. 15, 2197 (2013).

${ }^{45}$ A. Schäfer, C. Huber, and R. Ahlrichs, J. Chem. Phys. 100, 5829 (1994) 